\title{
Cave respiratorische Azidose beim Fliegen!
}

Hintergrund und Fragestellung: Patienten mit respiratorischen Erkrankungen können bei einer Flugreise aufgrund der hypoxischen und hypobaren Druckverhältnisse eine signifikante Hypoxie erleiden. Üblicherweise wird dies durch Sauerstoffgabe kompensiert. Es gibt aber bisher keine Empfehlung, was bei drohendem hyperkapnischem Atemversagen auf Flugreisen zu tun ist.

Kasuistik: Eine 76-jährige Frau mit respiratorischer Globalinsuffizienz wurde gemäß Empfehlung vor ihrer Flugreise mit einem Hypoxie-Simulationstest (Hypoxic Challenge Test $=$ HCT) untersucht, um die Flugsituation zu simulieren. Die klinisch stabile Patientin wurde dauerhaft nachts nicht invasiv beatmet. Die Blutgase waren stabil $\left(\mathrm{PaO}_{2} 9,12 \mathrm{kPa}, \mathrm{pH} 7,36, \mathrm{PaCO}_{2} 5,64 \mathrm{kPa}, \mathrm{O}_{2}-\right.$ Sättigung 98\%). Beim HCT wurde $15 \%$ iger $\mathrm{O}_{2}$ über Maske appliziert. Hierunter fiel die Sättigung auf $<85 \%$. Die Blutgasanalyse bestätigte dies $\left(\mathrm{PaO}_{2}<6,6 \mathrm{kPa}\right)$, sodass eine $\mathrm{O}_{2}$-Insufflation für eine Flugreise empfohlen wurde. Unter paralleler $\mathrm{O}_{2}$-Gabe von 2 1/min via Nasenkanüle wurde die Hypoxie überwunden. Der $\mathrm{PaCO}_{2}$ stieg aber auf 6,9 kPa,

\section{Originalie}

Spurling KJ, Moonsie IK, Perks JL. Hypercapnic Respiratory Acidosis During An In-Flight Oxygen Assessment. Aerosp Med Hum Perform 2016;87(2):144-7. es kam zur respiratorischen Azidose (pH 7,25). Im Ergebnis wurde der Frau dringend von Flugreisen abgeraten, da sie bei positivem $\mathrm{HCT}$ mit $\mathrm{O}_{2}$ eine ventilatorische Ateminsuffizienz mit respiratorischer Azidose entwickelte.

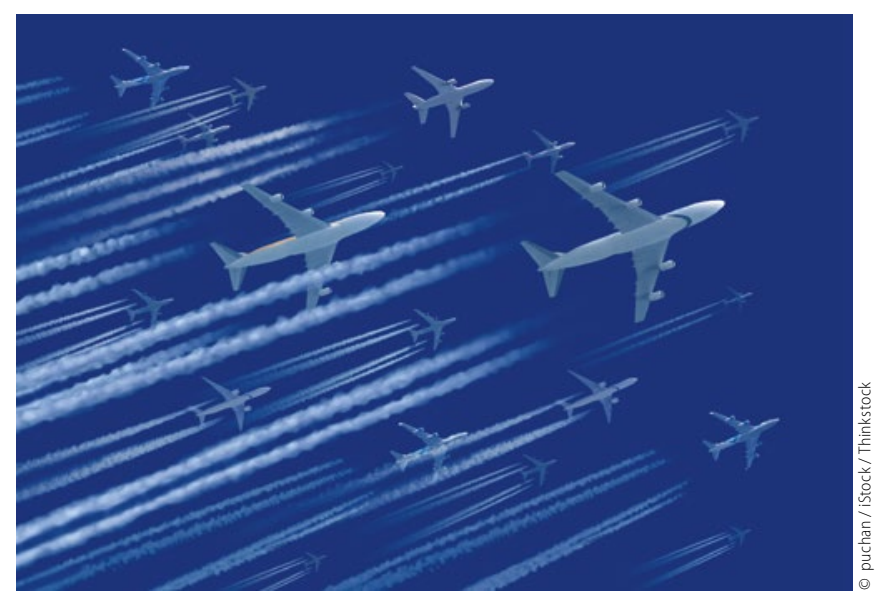

Mit dem Flugverkehr steigt auch die Zahl Lungenkranker in den Flugzeugen. Gut, wenn sie vorher kompetent beraten werden konnten.

Diskussion: Patienten mit bekannter globaler respiratorischer Ateminsuffizienz können in einer Flugsituation aufgrund des benötigten und substituierten Sauerstoffs eine respiratorische Azidose entwickeln. In diesen Fällen wäre eine Überprüfung der Blutgase zur Vermeidung einer Hyperkapnie sinnvoll. Bislang liegen keine Leitlinien vor, die das Risiko einer Hyperkapnie auf Flugreisen bei $\mathrm{O}_{2}$-Gabe berücksichtigen. Es wäre sinnvoll, dies in Studien zu untersuchen und Empfehlungen zu erarbeiten.

\section{- Kommentar von Dr. med. Christina Priegnitz}

\section{Mehr Know-how und Empfehlungen nötig}

In der Tat entsprechen die aktuellen Leitlinien zur Diagnostik vor einer Flugreise für pneumologische Patienten einem geringen Evidenzlevel [1] und werden in der Realität heterogen umgesetzt. Den Empfehlungen entsprechend wurde die Patientin zunächst pulsoximetrisch und blutgasanalytisch untersucht. Bei Unterschreiten der $\mathrm{O}_{2}$-Sättigung $<92 \%$ soll eine $\mathrm{O}_{2}$ Gabe folgen, bei Werten von 92-95\% ein 6-Minuten-Gehtest $[2,3]$. Bei Entsättigung unter $84 \%$ nach Gehtest wird eine $\mathrm{O}_{2}$ Gabe, $>84 \%$ der diagnostische Goldstandard, empfohlen: die Hypoxiesimulation mit einer reduzierten $\mathrm{O}_{2}$-Gabe $\left(15,1 \% \mathrm{O}_{2}\right)$ $[1,4,5]$, die einer Kabinenhöhe von 2438 m entspricht.

Die 76-Jährige erhielt daher aufgrund ihrer relevanten Hypoxie im $\mathrm{HCT}$ eine probatorische $\mathrm{O}_{2}-\mathrm{Gabe}$ von $2 \mathrm{l} / \mathrm{min}$. Dass dies bei ihr schon im normobaren Zustand zu einem respiratorischen Versagen mit respiratorischer Azidose führte, stellt die Schwere ihrer ventilatorischen Ateminsuffizienz dar.

Dieser Fall weist auf mehrere Punkte hin: Es bleiben Lücken in der sicheren Vorabtestung von pneumologischen Patienten vor Flugreisen. Auch bei Anwendung empfohlener Algorithmen werden nicht alle Fragen zur Vermeidung eines respiratorischen Versagens beantwortet. Dies kann nur zu weiteren klinischen Studien anregen. Bis dahin wird eine Empfehlung in Bezug auf eine Flugreise bei Lungenpatienten oft der klinischen Güterabwägung unterliegen.
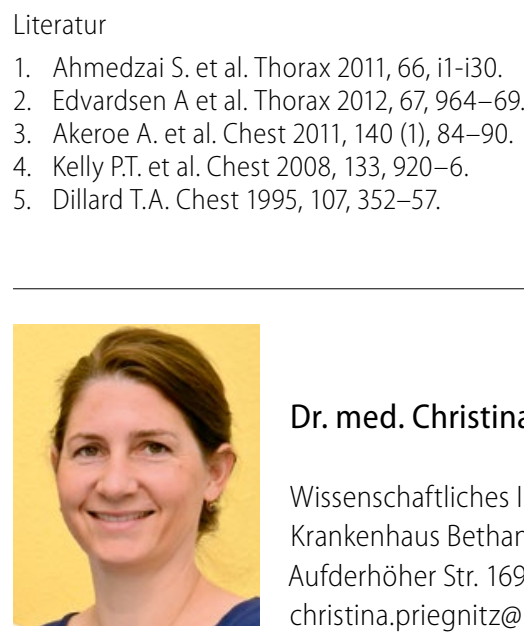

Dr. med. Christina Priegnitz

Wissenschaftliches Institut Bethanien e.V., Krankenhaus Bethanien gGmbH, Aufderhöher Str. 169-175, 42699 Solingen christina.priegnitz@klinik-bethanien.de 